\title{
Brain Mapping as a Tool to Study Neurodegeneration
}

\author{
Liana G. Apostolova and Paul M. Thompson \\ Department of Neurology and Laboratory of NeuroImaging, David Geffen School of Medicine, University of California-Los \\ Angeles, Los Angeles, California 90095
}

\begin{abstract}
Summary: Alzheimer's disease (AD) is the most common neurodegenerative disorder for those 65 years or older; it currently affects 4.5 million in the United States and is predicted to rise to 13.2 million by the year 2050 . Neuroimaging and brain mapping techniques offer extraordinary power to understand $\mathrm{AD}$, providing spatially detailed information on the extent and trajectory of the disease as it spreads in the living brain. Computational anatomy techniques, applied to large databases of brain MRI scans, reveal the dynamic sequence of cortical and hippocampal changes with disease progression and how these relate to cognitive decline and future clinical outcomes. People who are mildly cognitively impaired, in particular, are at a fivefold increased risk of imminent conversion to
\end{abstract}

dementia, and they show specific structural brain changes that are predictive of imminent disease onset. We review the principles and key findings of several new methods for assessing brain degeneration, including voxel-based morphometry, tensor-based morphometry, cortical thickness mapping, hippocampal atrophy mapping, and automated methods for mapping ventricular anatomy. Applications to $\mathrm{AD}$ and other dementias are discussed, with a brief review of related findings in other neurological and neuropsychiatric illnesses, including epilepsy, HIV/AIDS, schizophrenia, and disorders of brain development. Key Words: Brain mapping, MRI, dementia, mild cognitive impairment, Alzheimer's disease, schizophrenia, depression, human brain development.

\section{INTRODUCTION}

The possibility of creating maps that localize cognitive functions and disease-related changes to discrete brain regions has long fascinated the scientific world. Perhaps the most celebrated brain mapping achievement was Brodmann's cytoarchitectonic map, which in the early 20th century distinguished 52 cortical subregions based on their differences in thickness, lamination, neuronal type, and staining characteristics. ${ }^{1}$ Since the advent of noninvasive neuroimaging, brain maps have evolved to consist of sophisticated multimodal and multidimensional digital atlases that cover the human life span and represent a variety of diseases and their progression. ${ }^{2}$

Neurodegenerative diseases are a large group of neurologic disorders that share a similar pathophysiology. The vast majority are associated with the intracellular or extracellular deposits of abnormal proteins and those affected present with significant neurologic and cognitive dysfunction. ${ }^{3}$ The present review will focus mainly on the most prevalent neurodegenerative disorders, Alz-

Address correspondence and reprint requests to: Liana G. Apostolova, M.D., Alzheimer's Disease Research Center, 10911 Weyburn, 2nd floor, Los Angeles, CA 90095. E-mail: lapostolova@mednet. ucla.edu. heimer's disease (AD) and related dementias. We will also briefly summarize recent brain mapping advances in some other progressive neurologic and psychiatric disorders, such as schizophrenia, epilepsy, HIV/AIDS, and abnormal brain development.

Alzheimer's disease is the most common neurodegenerative disorder in those 65 years or older. It results from the pathological accumulation of two highly amyloidogenic proteins, A-beta and tau. ${ }^{3}$ It currently affects 4.5 million people in the United States, and this is projected to rise to 13.2 million by the year $2050 .{ }^{4}$

Clinically, $\mathrm{AD}$ is preceded by a transitional cognitive state called mild cognitive impairment (MCI), in which persons experience memory or other cognitive changes but continue to lead an independent lifestyle. ${ }^{5}$ As AD pathology builds up, cognitive decline relentlessly progresses. Autonomy in daily living becomes increasingly difficult, and most patients eventually become fully dependent on others for even the most basic activities of daily living, such as toileting or eating. The profound social, economic, and emotional burden of $\mathrm{AD}$ has brought $\mathrm{AD}$ research to the forefront as one of the most pressing health problems of the 21 st century. Currently available treatment options for AD patients are limited to symptomatic therapeutic agents, which have not con- 


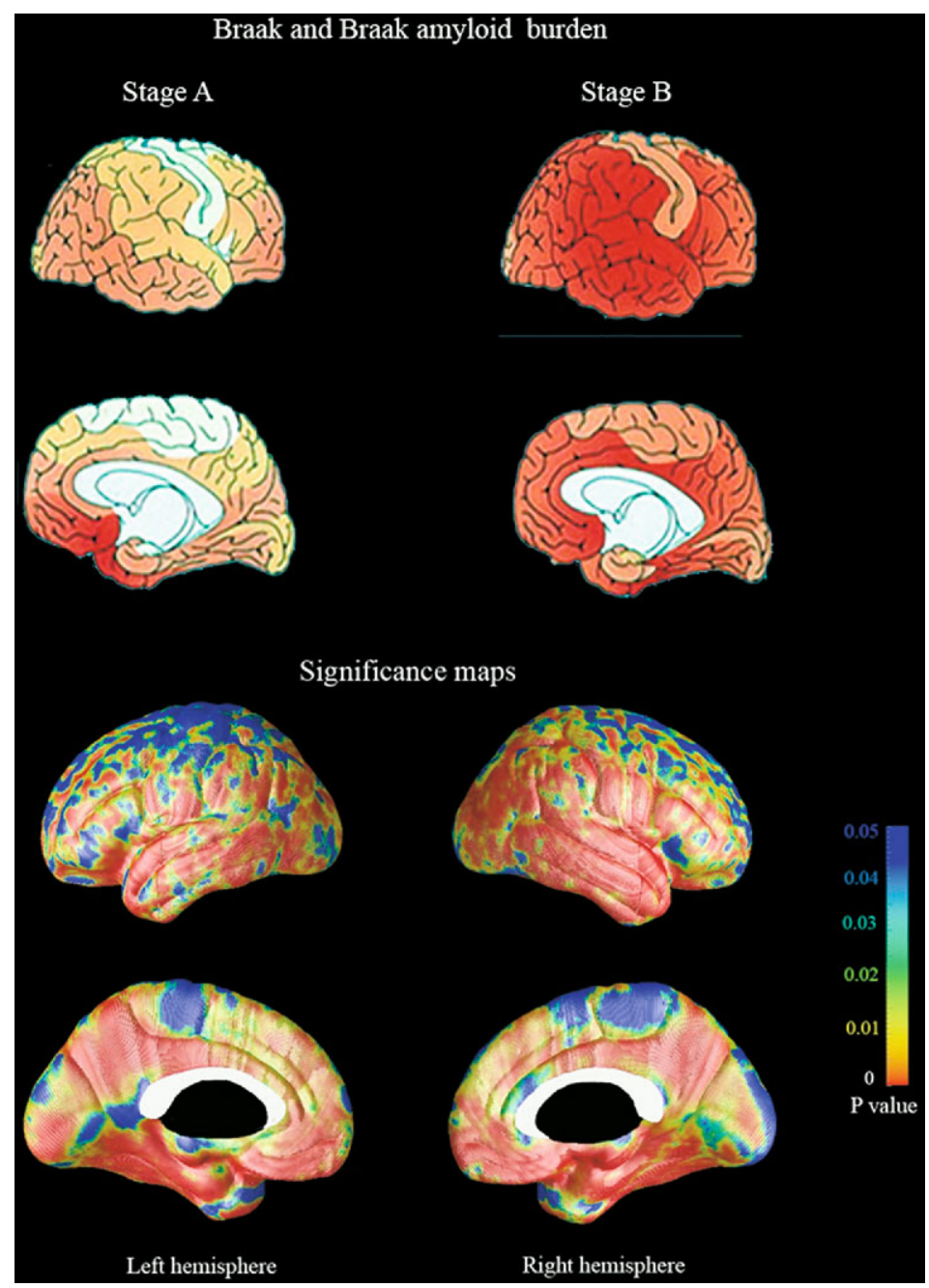

FIG. 1. A cross-sectional three-dimensional (3D) MRI study used the cortical pattern-matching technique to compare the gray matter density differences between mild Alzheimer's disease (AD) (mean Mini-Mental State Examination score MMSE $=23.8$ ) and amnestic mild cognitive impairment $(\mathrm{MCl})($ mean MMSE $=28.2) \cdot{ }^{71}$ The top half shows illustrations of Braak and Braak stages $A$ and $B$ for amyloid plaque burden. The bottom half shows the statistical comparison maps between the groups. Despite the small mean differences on a global cognitive measure (the MMSE), profound and widespread cortical atrophy is evident in mild AD versus amnestic MCl.

vincingly been shown to slow disease progression. With several disease-modifying agents at various stages of clinical testing, researchers are working in parallel to develop the most sensitive combinations of clinical and biologic markers for early or even preclinical diagnosis, and to track disease progression and neurotherapeutic effectiveness.

\section{CONVENTIONAL NEUROIMAGING TECHNIQUES AS DISEASE BIOMARKERS IN AD}

The earliest brain changes in AD occur in the entorhinal cortex and the hippocampus, where neurofibrillary tangles (NFTs) initially accumulate. These early events are followed by a spread of NFTs to the inferior and lateral temporal, then parietal, occipital, and finally frontal cortices. The amyloid neuritic plaques originate in the lateral temporal neocortex and then spread to the inferior temporal, parietal, occipital, and frontal association cortices $^{6,7}$ (FIG. 1, top).

As the earliest sites to accumulate NFT pathology, the hippocampus and entorhinal cortex have become the most studied brain regions in AD. They have been traditionally examined in structural brain images with a region-of-interest (ROI) technique. ROI techniques typically compute an overall volume for each brain structure, based on manual or automated delineations in serial sections of a subject's MRI.

To ensure adequate reliability in defining the structure boundaries, a formalized anatomical protocol is typically used, with detailed rules to guide image analysts in segmenting the structure with high reliability and consistency across raters. Automated, computer vision algorithms have also been developed to delineate these 
structures automatically on MRI, but none of these are as yet widely used. ${ }^{8-10}$ Reliable automated segmentations are difficult to obtain in the medial temporal lobe, because anatomical boundaries are complex and image contrast is often low and is even affected by the disease process. The a priori knowledge that these structures are involved in MCI and AD makes ROI-based analysis a powerful approach, but not one that permits detailed investigation of the underlying complex structure of the hippocampus. Nevertheless it was through this technique that the hippocampus and entorhinal cortex were established as the most prominent imaging biomarkers in AD.

Hippocampal atrophy occurs in normal aging at an estimated rate of $\sim 1.6-1.7 \%$ annually. ${ }^{11,12}$ In MCI and $\mathrm{AD}$, the yearly atrophy rates are severalfold higher $(2.8 \%$ for stable MCI, $3.7 \%$ for MCI who convert to AD, and $3.5-4 \%$ for AD subjects). ${ }^{11,12}$ Additionally, smaller average hippocampal volume in MCI carries an increased risk (odds ratio of 1.75) for future conversion to $\mathrm{AD}$ over a follow-up period of $1.2-4.8$ years. ${ }^{13}$ Similarly, in genetically predisposed AD patients with autosomal dominant mutations, progressive hippocampal atrophy is detected as much as 5.5 years before clinical diagnosis of AD can be established. ${ }^{14}$

As useful a simple measure as regional volume can be, it nonetheless does not adequately capture the complex profile of disease progression, which involves selective changes in certain hippocampal subfields. From the entorhinal cortex, NFTs spread first to the CA1 (cornu ammonis 1) and subiculum, then to the CA2/3 and finally to the CA4 hippocampal subfield. ${ }^{15}$ Newer techniques (discussed under Brain mapping as a tool to study neurodegeneration, the next section) can now better localize discrete subregional changes that can predict disease progression in MCI. Subregion-specific changes between $\mathrm{AD}$ and MCI can also be related to known cytoarchitectural subdivisions. $^{16,17}$

The entorhinal cortex has likewise been extensively studied with ROI techniques, and its predictive value for future cognitive decline in MCI is well established. ${ }^{18-21}$ The annual rate of $1.4 \%$ entorhinal cortical atrophy in normal aging is far surpassed by the pathological $7 \%$ average annual loss observed in AD. ${ }^{22}$ The ROI technique is well suited for the simple sheet-like structure of the entorhinal cortex; however, significant technical challenges stem from its substantial anatomical variability, ambiguous boundaries, and the lack of a widely embraced tracing protocol-this last making betweenstudy comparisons challenging. A major limitation of the ROI approach for the entorhinal cortex lies in its focus on one important but small area of the brain in a neurodegenerative disease that affects the cortex in its entirety.

Voxel-based morphometry (VBM) is a newer image analysis technique that can simultaneously identify multiple areas of cortical and subcortical degeneration. It has provided significant insights into the gray matter changes in $\mathrm{AD}$ and, more recently, also in MCI. Initially applied to studies of schizophrenia, ${ }^{23} \mathrm{VBM}$ was subsequently implemented in the widely used Statistical Parametric Mapping software package (Wellcome Department of Imaging Neuroscience, University College London, London, UK). ${ }^{24-26}$

The standard VBM method classifies each subject's three-dimensional (3D) brain MRI scan into individual maps that are representative of gray and white matter and CSF tissue classes. The gray matter images from all subjects are then aligned to a common template before the results are averaged. The intensity of these spatially normalized gray matter maps is spatially smoothed with a filter, ${ }^{24,27,28}$ and group differences or associations with cognitive scores are assessed by fitting a statistical model (typically multiple regression) at each image location. Statistical differences are then shown as a spatial map.

The most common statistical analysis in VBM fits the general linear model (GLM) to the data (gray matter density) from all subjects at each image location, or voxel. This identifies voxels where tissue density relates to covariates of interest (e.g., diagnosis or cognitive scores) after discounting confounding effects (e.g., age, sex, or educational level). As in more traditional volumetric studies, a measure of total gray matter or overall cerebral volume may be used as a covariate of interest, to detrend brain size or global atrophy effects from the data. Including the overall amount of gray matter as a covariate in the model enables detection of gray matter that is regionally specific, beyond any global differences.

Some caveats are required in interpreting the findings of VBM, because it can infer local anatomical differences 1) from systematic image registration errors in one group relative to the other and 2) from systematic shifts in unaffected regions that result from differences in truly affected structures. ${ }^{29,30}$ In response to such criticisms, the VBM authors improved the method to better reflect volumetric differences between subjects, and to avoid incorrect localization of group differences that can occur due to imperfect registration of the images. ${ }^{25}$

With the original VBM approach, comparisons of gray matter density alone across subjects do not fully reflect volume differences between subjects, because they do not contain information on the expansions and contractions needed to match the subject to the common template. The modulated, or optimized, VBM method ${ }^{27}$ addresses this issue by multiplying (modulating) the voxel intensity values of the spatially normalized gray matter maps by the corresponding Jacobian determinant (expansion factor) of the deformation fields, so that the total amount of gray matter at each voxel, which is the product of the gray matter density and the volume of the deformed voxel, remains unchanged during warping. The method then reveals systematic volume differences for 
any brain region, assuming the warping process matches anatomy correctly across subjects. A conceptually similar approach (RAVENS) has been proposed by Davatzikos et al. ${ }^{31}$ and has been used successfully in studies of degenerative disease.

VBM still has some limitations, stemming from the inherently low spatial resolution caused by spatial smoothing of the gray matter maps in order to control for interindividual cortical variability. Governed by the full width at half-maximum of the smoothing kernel (most commonly $12 \mathrm{~mm}$; see Salmond et al. ${ }^{32}$ for different choices), the spatial resolution of VBM is limited. By the matched filter theorem, larger smoothing filters make it easier to detect diffuse or widespread effects, at the expense of blurring observations from different anatomic regions. Smoothing 1) partly accounts for registration errors and reduces interindividual variance, increasing detection sensitivity, and 2) makes the data a better approximation to a Gaussian random field. This normality of the residuals is a requirement if parametric statistics are used in later processing.

Several VBM studies have documented gray matter atrophy in the temporal, posterior cingulate, and precuneal cortex in AD relative to normal controls. ${ }^{33-36}$ As expected, these differences are less profound between $\mathrm{MCI}$ and $\mathrm{AD}$ and between MCI and normal aging. Thus, in agreement with clinical observations that place MCI between normal cognitive aging and $\mathrm{AD}$ based on atrophy severity, MCI likewise appears to be intermediate between normal aging and AD. ${ }^{37-39}$ Progressive brain atrophy has been documented in MCI subjects who have declined (and even in those who have remained stable), providing evidence for a neurodegenerative etiology and advancing disease pathology in the latter as well. ${ }^{40}$ Whole brain size and ventricular size are powerful predictors of future cognitive decline throughout the spectrum of normal aging to onset of $\mathrm{AD} .{ }^{13}$

The predictive role of VBM was persuasively demonstrated in a study of 28 asymptomatic carriers of dominantly inherited genetic forms of early-onset AD. Subjects who converted to dementia had an annual brain volume loss of $5 \%$, compared with only $0.1 \%$ in those who remained cognitively intact. ${ }^{41}$ Ventricular enlargement appears to have a higher predictive value in the earlier transition stages as opposed to the later stagethat is, from normal cognition to $\mathrm{MCI}$ or $\mathrm{AD}$ and less so from MCI to AD. ${ }^{13,42}$ VBM has also been used to reveal the spatial profile of aging and gender effects in normal subjects, ${ }^{28,43,44}$ frontotemporal and Lewy body dementia, ${ }^{45-48}$ Parkinson's disease, and even herpes simplex encephalitis. $^{49}$

For analysis of cortical gray matter atrophy, VBM may not be optimal. Gyral and sulcal features are highly convoluted, and individual gyri or sulci are not readily distinguished based on image intensity alone. As a result, most automated image warping algorithms, relying on image intensities to match anatomical features, fail to match cortical gyri across subjects in morphometric comparisons. This leads to a lack of power in detecting and localizing subtle cortical differences. In response, surface-based modeling methods have used specific cortical features as constraints to guide the matching of anatomy across subjects. Some of these methods also compute the cortical gray matter thickness directly in three dimensions based on an explicit model of the cortical surface geometry or on successive coding of voxels within the gray matter sheet. ${ }^{50,51}$

\section{BRAIN MAPPING AS A TOOL TO STUDY NEURODEGENERATION}

Several powerful brain mapping techniques have emerged since the mid-1990s. Many rely on computational anatomy, a mathematical brain modeling approach in which brain surfaces and subvolumes are viewed as complex geometrical patterns and are modeled as 3D continuous mesh models (or deformable shapes) that can be averaged and combined across subjects, and on which statistics can be defined. (Gee and Thompson ${ }^{52}$ have provided a recent review of the field.) The anatomical images thus represent deformable templates that are elastically or fluidly transformed into a similar shape, most commonly onto the study group average, an atlas average, or another subject's brain shape.

Some of these techniques explicitly model brain anatomy; for instance, they may use surface landmarks as constraints (e.g., sulci). These techniques readily allow for an accurate alignment of surface specific geometrical patterns (e.g., gyri) and help to precisely colocalize identical cortical and subcortical regions without sacrificing the underlying details contained in the measure of interest (e.g., cortical thickness, gray matter density, or functional activation) (FIGS. 1 and 2). The resulting anatomical coregistration increases the power to characterize cortical and subcortical disease patterns and detect subtle disease-associated changes.

One newer technique to localize changes within the brain is known as tensor-based morphometry (TBM). TBM relies on a fully automated fluid warping to spatially register cortical and subcortical structures in crosssectional and longitudinal imaging datasets (see Chiang et al. ${ }^{53}$ for mathematical details). When TBM is used to map brain changes over time, the follow-up (repeat) image is first globally aligned to the baseline scan, and then a 3D elastic or fluid image deformation is used to maximize the mutual information (or a related information-theoretic measure of correspondence) between the two consecutive scans. This fully 3D deformation reconfigures the baseline anatomy into the shape of the follow-up scan. The expansion or contraction at each image 


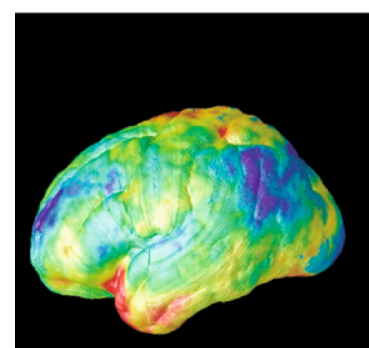

Left hemisphere

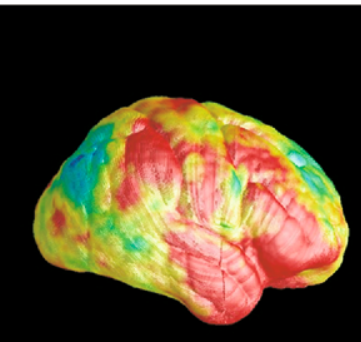

Right hemisphere

\section{BASELINE}

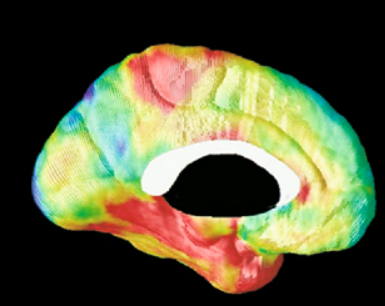

Left hemisphere

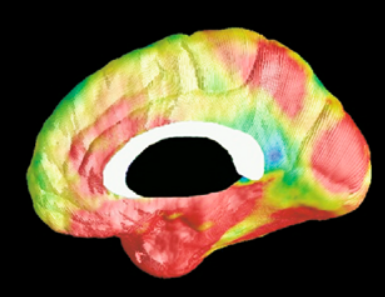

Right hemisphere

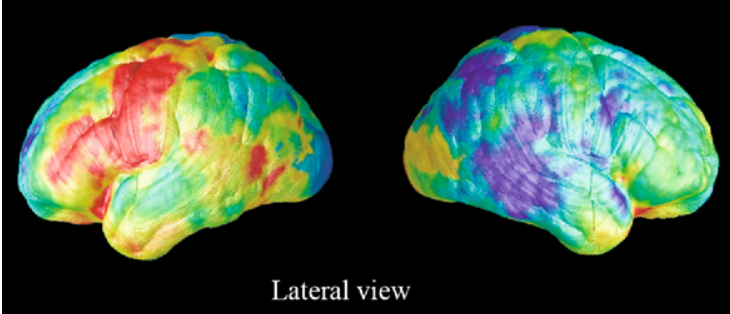

Lateral view

\section{FOLLOW-UP}
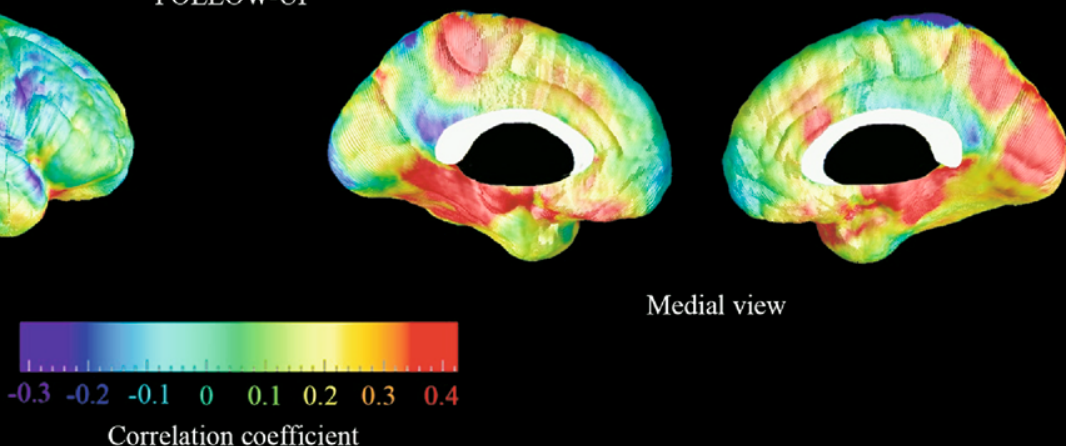

Medial view

Correlation coefficient

FIG 2. This longitudinal 3D $\left[{ }^{18} \mathrm{~F}\right]$ fluorodeoxyglucose PET study investigated changes in brain metabolism in 6 cognitively normal elderly and $16 \mathrm{MCl}$ subjects over a 21-month period. At follow-up, two normal subjects were diagnosed with $\mathrm{MCl}$, and two additional subjects (one $\mathrm{MCl}$, and one normal, initially) had converted to $\mathrm{AD}$. The remaining 15 subjects remained stable. Shown is the correlation between relative hypo- or hypermetabolism and cognitive outcome (showing groups who had deteriorated or remained $\mathrm{MCl}$ versus normal controls at baseline and follow-up). Metabolic differences at baseline are seen bilaterally in the entorhinal, inferior temporal, precuneal, right posterior dorsolateral frontal, and anterior cingulate cortices. Relative hypometabolism was seen in the left parietal and anterior frontal cortices. At follow-up, the hypermetabolic areas were greatly reduced or completely abolished. A new area of relative hypermetabolism was evident in the left posterior frontal cortices. These findings suggest the interval loss of compensatory hyperactivation in subjects predestined to cognitive decline (unpublished data).

voxel is computed from the deformation field (using the Jacobian of the deformation field to produce a voxel compression map or tensor map). In this map, contraction implies atrophy; expansion implies local growth or dilation. ${ }^{54}$ A color map then displays these changes on the follow-up scan (FIG. 3).

Brain changes can be mapped in patients scanned longitudinally, such as individuals with semantic dementia, ${ }^{55}$ or normal subjects scanned over short intervals to assess longitudinal stability of a scanning protocol. ${ }^{55}$ TBM has high throughput and sensitivity, making it attractive for gauging brain changes in large population studies and clinical trials. TBM may also be used for cross-sectional studies; in that case, many individual images are fluidly transformed to match a common brain template, and the applied expansions and contractions are analyzed to identify systematic volume and shape differences in one subject group versus another. Used

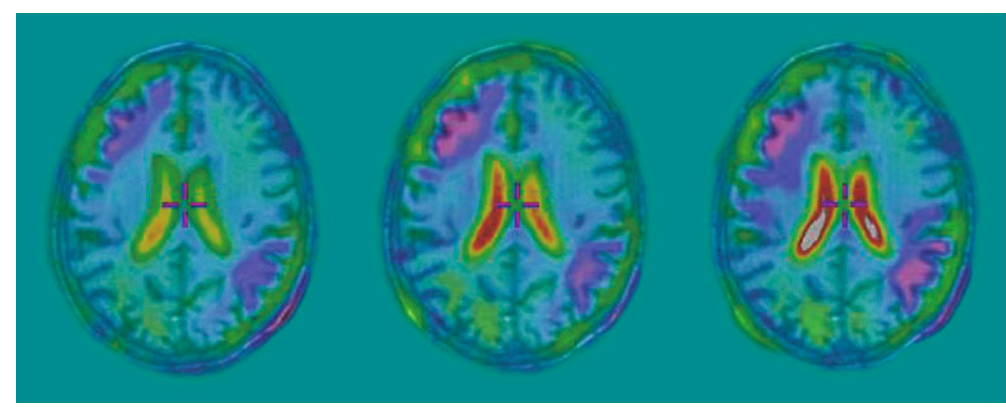

FIG. 3. These maps show progressive brain changes in a patient with posterior cortical atrophy, based on tensor-based morphometry. Blue and purple colors denote areas of progressive volume loss in the frontal and parietal cortices, based on a comparison of brain MRI scans taken 1, 1.5, and 2 years after initial diagnosis. Red and white colors illustrate the progressive ventricular enlargement. The color-coded maps (Jacobian maps), show the local expansion or compression factor required to fluidly align baseline to each follow-up scan, after they are initially aligned with a rigid-body transformation. These maps may also be averaged voxel-by-voxel across subjects or compared across populations to assess factors that influence degenerative rates in each region of the brain. 


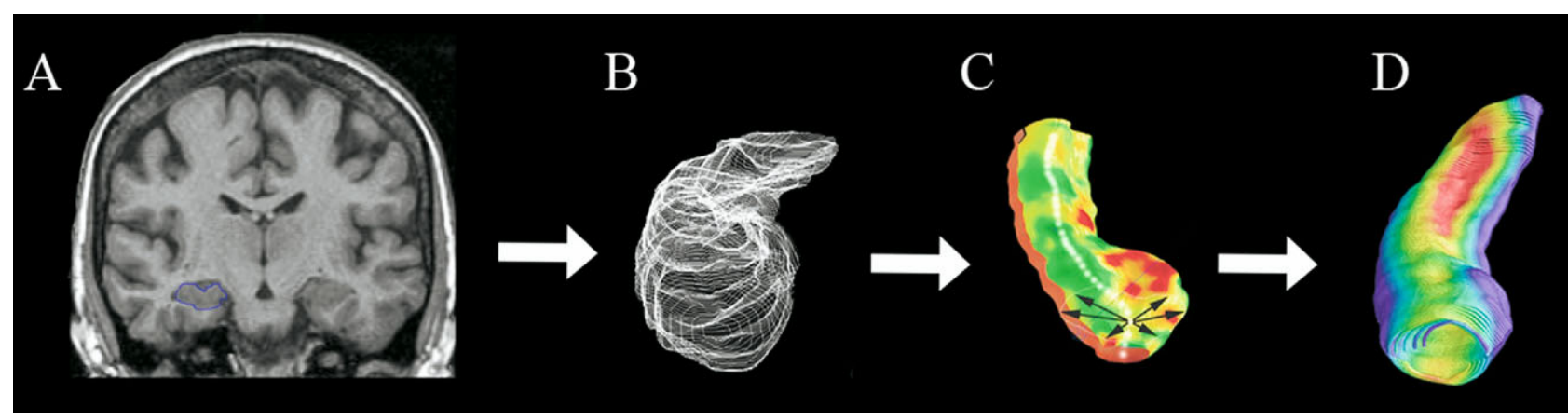

FIG. 4. The radial distance method relies on manual tracing of the hippocampus $(A)$ followed by the computation of a $3 D$ parametric mesh model of the structure (B), estimation of the distance between the central core of the structure to each surface point (e.g., radial distance) (C), recording of the radial distance estimates at each surface point and production of individual and group average (D) color-coded maps of the radial distance that are statistically compared between groups and/or conditions.

cross-sectionally, TBM has provided powerful visualizations of brain deficits, and their clinical correlates, in populations with HIV/AIDS, ${ }^{53}$ fragile $\mathrm{X}$ syndrome, ${ }^{56}$ and Williams syndrome. ${ }^{57}$

New statistical methods are also emerging to make TBM more sensitive to subtle neurodegenerative changes. If only the compressions and expansions are analyzed, then most of the information on atrophy, which may be directionally dependent (i.e., nonisotropic), is discarded. The so-called Lie group methods can detect neurodegeneration with greater power (e.g., in studies of HIV/AIDS ${ }^{58}$ ), because they draw on the full multidimensional information available in the deformation tensors and model the tensor statistics as a statistical process on a Riemannian manifold (an approach called generalized $T B M$ ). Thus, TBM can detect and visualize focal areas of structural gray and white matter and subcortical nuclear changes. $53,55,58$

\section{BRAIN MAPPING IN NEURODEGENERATIVE DEMENTIAS}

\section{Alzheimer's disease}

The 3D modeling techniques have enabled the localization of disease-associated changes at the subfield level of the hippocampus. Some of these techniques have relied on radial measurements that assess the thickness of a structure (FIG. 4), ${ }^{59}$ others on large-deformation highdimensional techniques ${ }^{60}$ or on fluid-based registration of structural data within or across subjects. ${ }^{42,61}$

Rather than building 3D average surface meshes from manually derived traces of the hippocampus, the highdimensional modeling approach uses an elastic registration method to deform a hippocampal surface template onto the scans of new subjects, adapting to anatomical shape differences. In this way it is a variant of the TBM approach, which uses fluid registration to measure volume change, and the hippocampal radial atrophy technique, which uses the surface models to measure local changes on the hippocampus. Aiming to detect substruc- tural changes within the hippocampus, our research group reported a technique for unfolding of the hippocampal gray matter sheet with subsequent alignment of the hippocampal subfields. ${ }^{62}$ Another research group delineated the hippocampal subfields on T2-weighted MRI data obtained at $4 \mathrm{~T}$ on three hippocampal slices and studied the effects of aging on the derived partial subfield volumes. ${ }^{63}$ Ultimately, the best approach to delineate hippocampal subfields in vivo may require higher-field MRI - at field strengths as high as $7 \mathrm{~T}$-in which the layers of the entorhinal cortex can be visualized. ${ }^{64}$

Neuropathological studies describe hippocampal NFT pathology as a stage-like process affecting the subiculum and CA1 subfields first, followed by the CA2/3 and finally the CA4 subfields. ${ }^{15}$ In 2004, Thompson et al. ${ }^{59}$ applied a hippocampal radial atrophy mapping technique to $\mathrm{AD}$ and normal elderly subjects, which revealed profound differences between the two groups. Using the same technique, Frisoni et al. ${ }^{65}$ demonstrated that the CA1 area and parts of the subiculum show 15-20\% atrophy in $\mathrm{AD}$ relative to normal controls. Apostolova et al. ${ }^{17}$ showed that $\mathrm{MCI}$ individuals with more severe CA1 and subiculum involvement are more likely to convert to $\mathrm{AD}$ (FIG. 5). Atrophy in the subiculum and CA1 area in this study also showed strong correlation with impaired delayed recall on the California Verbal Learning Test. ${ }^{17}$ In a follow-up study, Apostolova et al. ${ }^{16}$ compared the hippocampal data of MCI and AD subjects. In agreement with neuropathological data, the hippocampal formation in $\mathrm{AD}$ was found to be more involved, with greater atrophy of the CA1 and additional atrophy of the CA2 and CA3 subfields. ${ }^{16}$

Becker et al. ${ }^{66}$ also used the hippocampal radial atrophy technique in a cross-sectional study of single-domain amnestic MCI, multiple-domain amnestic and nonamanestic MCI, AD, and normal elderly subjects. The AD and single-domain amnestic MCI groups showed significant subicular atrophy relative to normal controls. The AD group had involvement of the CA1 


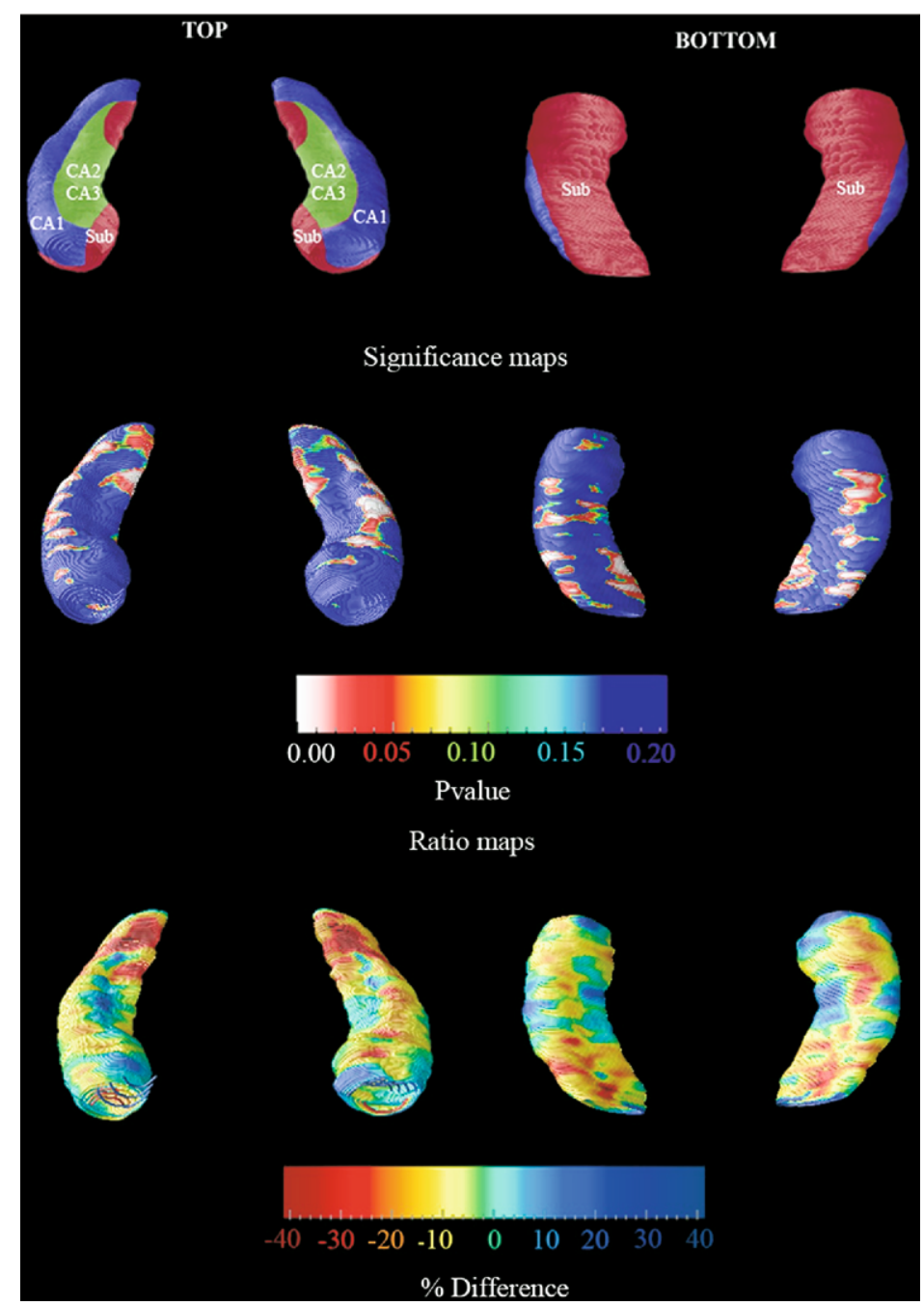

FIG. 5. This longitudinal $3 D \mathrm{MRI}$ study ${ }^{17}$ compared the baseline hippocampal radial atrophy of MCI subjects who converted to $A D$ and $\mathrm{MCl}$ subjects who remained stable or improved cognitively during the 3 years of clinical follow-up. The top row shows a schematic representation of the hippocampal subfields based on two established sources. ${ }^{105,106}$ The middle row shows the statistical comparison maps and the bottom row shows the absolute difference maps (in \%) in the mean radial distance estimates between these two groups. More atrophy at baseline is seen in the $\mathrm{CA} 1$ and subicular areas in $\mathrm{MCl}$ subjects who subsequently convert to clinically probable AD.

subfield as well. In contrast, the multiple-domain MCI group did not differ from the normal control group in either volumes or 3D hippocampal maps. ${ }^{66}$ Similar findings have been reported by researchers using the related large-deformation high-dimensional modeling approach. With this technique, two studies compared mild $\mathrm{AD}$ subjects with a Clinical Dementia Rating Scale of 0.5 to age-matched controls. They demonstrated predominant lateral edge hippocampal atrophy corresponding to the CA1 subfield. ${ }^{60,67}$

Apolipoprotein E4 (ApoE4) is the most prominent genetic risk factor for sporadic AD. A longitudinal study of cognitively normal elderly who were genotyped revealed that ApoE4 carriers had higher hippocampal atrophy rates relative to noncarriers. ${ }^{68}$ In a cortical thickness study, Bookheimer et al. ${ }^{69}$ reported that cognitively normal ApoE4 carriers have significantly thinner entorhinal cortex and focal hippocampal atrophy limited to the subiculum, relative to cognitively normal noncarriers.

The cortical pattern-matching technique ${ }^{70}$ is an advanced computational anatomy imaging methodology that uses sulcal constraints while elastically deforming each subject's cortical morphology into the group average representation. Cortical surface models, derived from structural MRI data, may be used to display a wide variety of imaging data, such as cortical thickness, gray matter density, functional activation data, or metabolic data from PET scans.

In the first published study using this technique, Thompson and colleagues compared 12 AD (Mini-Mental State Examination score, MMSE, at baseline $=18$, MMSE at 1.5 year follow-up $=14$ ) and 14 age-matched cognitively intact subjects, scanned twice 1.5 years apart. ${ }^{70}$ The baseline gray matter density comparison 
maps demonstrated significant $(>15 \%)$ atrophy in the lateral temporal, parietal and parieto-occipital cortices of $\mathrm{AD}$ patients. At follow-up, in agreement with the wellestablished disease progression sequence, these changes were shown to engulf the frontal lobes. On the mesial surface, profound atrophy was noted for the left hemisphere at baseline but the right was relatively preserved, with more focal precuneal and mesial temporal atrophy at baseline that spread to the cingulate cortex at followup. A time-lapse video sequence of the dynamics of $\mathrm{AD}$ spreading through the neocortex is available at http:// www.loni.ucla.edu/ thompson/AD_4D/dynamic.html.

Computational anatomy techniques may offer greatly improved sensitivity for detection of disease-induced group differences. A recent study compared amnestic MCI and mild AD subjects (FIG. 1). ${ }^{71}$ Despite the small cognitive differences between the two groups (mean MMSE difference of 4.4), the authors reported highly significant greater cortical atrophy in mild $\mathrm{AD}$ versus MCI. Most affected were the entorhinal, inferior and lateral temporal, and the medial parietal/posterior cingulate cortices, followed by the lateral parietal, occipital, and finally the frontal association cortices. This pattern concurs exceptionally well with the neuropathologic evidence for cortical disease progression (FIG. 1). ${ }^{6,7}$

Using an alternative cortical thickness mapping approach, Lerch et al. ${ }^{72}$ compared 19 subjects with mild to moderate $\mathrm{AD}$ (mean MMSE $=21.2$, range 10-29) with 17 healthy volunteers. The AD patients had globally thinner cortical mantles $(3.1 \pm 0.28 \mathrm{~mm}$ versus $3.74 \pm$ $0.32 \mathrm{~mm}$ in the controls). Statistically, the most profound differences were seen in the inferior and lateral temporal, precuneus, posterior cingulate, and temporo-occipital association cortices. ${ }^{72}$ The reported regional effects were similar to those reported by Apostolova et al. ${ }^{71}$ in the study comparing mild AD (mean MMSE $=23.8$ ) to MCI patients (mean MMSE $=28.2$ ).

A larger follow-up study included 34 healthy controls, 62 MCI subjects, and $42 \mathrm{AD}$ patients. ${ }^{73}$ This study revealed that, as predicted, MCI patients have intermediate cortical thickness values relative to normal controls and AD subjects (normal controls versus $\mathrm{MCI}$ mean cortical thickness difference $=0.18 \mathrm{~mm}$; MCI versus $\mathrm{AD}$ mean cortical thickness difference $=0.26 \mathrm{~mm}$ ). ${ }^{73}$ In comparisons of MCI versus normal controls, the strongest effect sizes were seen for the entorhinal and lateral occipitotemporal gyri. In the $\mathrm{AD}$ versus normal controls comparison, significant differences were seen throughout the lateral surface of the brain with relative sparing of the primary sensorimotor and occipital regions. Differences for the mesial hemispheric surface were not reported. ${ }^{73}$

A very recent cortical pattern-matching study ${ }^{74}$ examined the difference in severity and localization of cortical atrophy in subjects with sporadic early-onset $\mathrm{AD}(<65$ years of age) versus late-onset $\mathrm{AD}$ ( $>65$ years of age).
The early-onset subjects showed widespread atrophic changes relative to cognitively intact age-matched controls, but late-onset subjects showed a less severe and more focal pattern of entorhinal, parahippocampal, inferior temporal, posterior cingulate/precuneal, and lateral temporal changes. These findings imply that age of onset has a powerful influence on the severity of disease-induced changes required to produce cognitive symptoms. Younger AD subjects displayed higher tolerance to pathological burden, suggesting that they may have higher cognitive reserve.

Cortical neurodegeneration correlates with cognitive decline. To date, our research group has reported correlations for the MMSE, a global measure of cognitive function, ${ }^{5,76}$ for a verbal fluency test and a picture naming test (animal fluency and Boston Naming test), ${ }^{77}$ and for delayed recall of verbal information (California Verbal Learning Test). ${ }^{78}$ Strong associations between MMSE and cortical atrophy were seen in the entorhinal, parahippocampal, precuneus, superior parietal, and subgenual cingulate/orbitofrontal cortices. ${ }^{70,75}$ Impaired picture naming and semantic fluency correlated with atrophy in perisylvian cortical areas thought to house lexical phonologic and semantic representations and process the receptive and articulatory aspects of language. ${ }^{77}$ Delayed recall performance was associated with bilateral precuneal, entorhinal, parahippocampal, inferior temporal, and left temporo-occipital cortical atrophy. These areas have been implicated in retrieval of verbal information and storing of lexical phonologic representations. ${ }^{78}$

Some ROI-based and VBM studies have suggested that ventricular volumes and rates of expansion are sensitive markers for $\mathrm{AD}$ and even more so for MCI progression. ${ }^{13}$ Our research group recently developed a computational anatomy technique for fluid registration of parametric mesh models of the lateral ventricles to individual untraced ventricular volumes. To reduce error, ventricles are first traced by hand on a small subset of scans. The results of fluidly mapping these surfaces onto new unlabeled scans are averaged together, greatly reducing segmentation error within each scan. This technique, followed by the radial atrophy mapping approach used to study hippocampal atrophy, rapidly and fully automatically assesses ventricular expansion in neurodegenerative disorders (FIG. 6). ${ }^{61}$ Studies are currently under way to define ventricular changes in subjects with MCI and AD. A similar approach was used by Carmichael et al., ${ }^{42}$ who reported that among normal subjects larger ventricles at baseline were predictive of future progression to dementia.

These automated surface mapping methods offer the high-throughput required for population-based studies or clinical trials; for example, 339 subjects were automatically mapped in a recent cohort from the Cardiovascular Health Study. ${ }^{42}$ These methods provide maps of statistics 


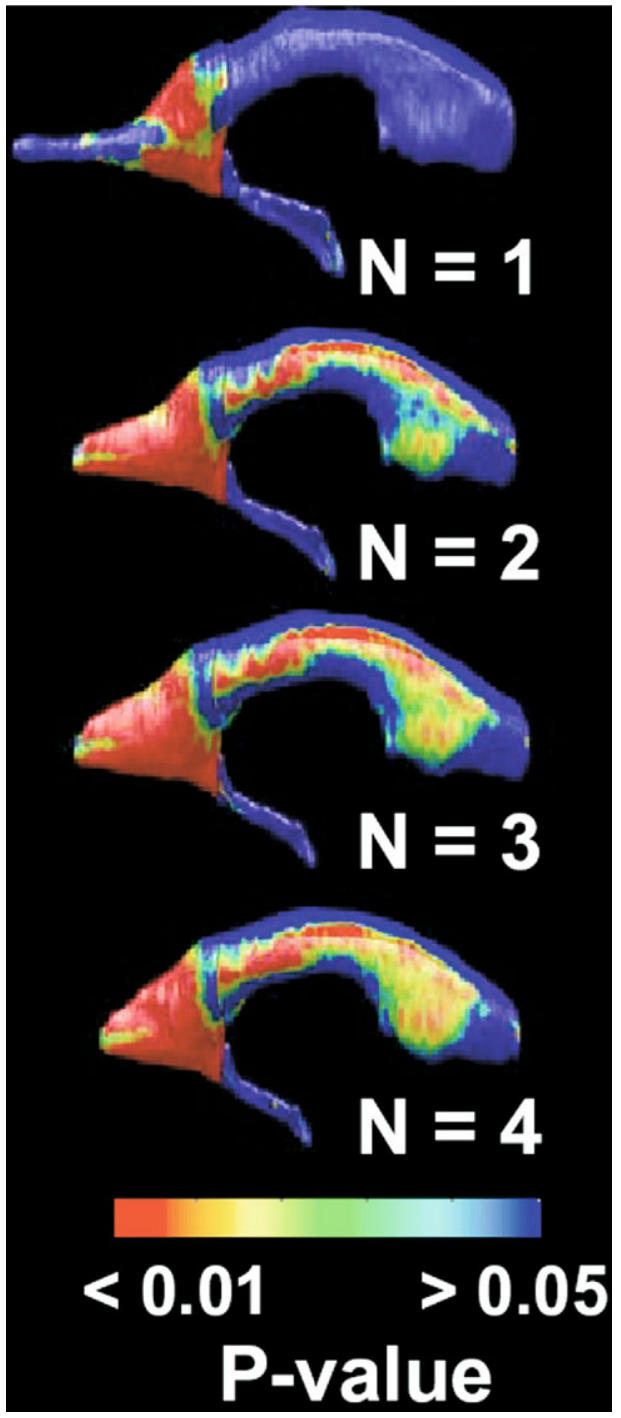

FIG. 6. This cross-sectional $3 D$ ventricular study validated the recent fluid registration method that adapts single-subject derived parametric mesh models of the lateral ventricles to individual untraced ventricular volumes. ${ }^{61}$ This figure shows the incremental gain in statistical power and decremental reduction of segmentation error when up to four individual parametric mesh primers are used to label the ventricles in each new scan followed by shape averaging of the resulting models. This approach is then followed by the radial atrophy mapping technique to show the areas of statistically significant expansion in the ventricular shape (in yellow to red) in an AD versus a normal control group.

that are often better able to detect subtle or localized atrophy than traditional numeric summaries. Even so, clinical trials often require neuroimaging outcomes to be expressed in terms of a simple set of numeric summaries (rather than a 3D map, for example). Current efforts are therefore also focused on deriving single numeric summaries from the brain maps that optimally predict conversion from $\mathrm{MCI}$ to $\mathrm{AD}$, or therapeutic response in large patient cohorts assessed with longitudinal MRI. ${ }^{79}$

\section{Other dementias}

Dementia with Lewy bodies (DLB) is the second most common dementing disorder affecting the elderly. Patients with DLB frequently show several of the following cardinal features: progressive cognitive decline, earlyonset hallucinations and delusions, Parkinsonism, and a fluctuating course. The pathologic hallmarks for DLB are synuclein-rich intracellular deposits known as Lewy bodies. Frequently, Lewy body and AD-type pathology coincide; when they do, patients may present with a clinical picture closely resembling $\mathrm{AD}$, which makes it difficult to differentiate the two disorders on clinical grounds alone.

A VBM study by Whitwell et al. ${ }^{48}$ recently reported a distinct cortical atrophy pattern in DLB that may help differentiate between DLB and AD, the two most common dementias: namely, hippocampal and inferior temporal preservation along with midbrain atrophy in DLB. Using the cortical pattern-matching technique, Ballmaier et al. ${ }^{80}$ demonstrated that preservation of the temporal and orbitofrontal cortices in demented subjects is suggestive of DLB, as opposed to AD.

Frontotemporal dementia (FTD) is a spectrum of disorders that typically affects younger patients than other dementias do. The behavioral variant (also known as frontal variant FTD, or fvFTD) is characterized by progressive atrophy of the frontal lobes ${ }^{81}$ and affects middle-aged adults, whereas the two temporal variants (primary progressive aphasia and semantic dementia) are characterized by progressive language impairments, a predominant left temporal atrophy pattern ${ }^{82}$ and a tendency to affect middle- to older-aged adults. Two preliminary studies using surface-based computational anatomy techniques demonstrated a frontal and right-sided predilection of fvFTD versus posterior and left-sided predilection of semantic dementia ${ }^{83}$ and the classic frontal FTD versus the classic posterior $\mathrm{AD}$-associated cortical atrophy pattern. ${ }^{84}$

\section{STRUCTURAL BRAIN MAPPING IN OTHER NEUROLOGICAL DISORDERS}

Epilepsy is a chronic neurologic disorder that affects humans throughout their life span. The main characteristics are episodic spontaneous synchronous neuronal discharges that clinically manifest as seizures. It is another neurological disease with enormous social and emotional impact. The commonest variant is mesial temporal lobe epilepsy (MTLE), caused by aberrant neuronal circuitry most often localized to the hippocampus and/or the amygdala. When therapy-resistant and intractable, MTLE is frequently surgically amenable through a procedure known as anterior temporal lobectomy. However, extreme caution, precise diagnosis, and localization of the seizure focus are imperative before any patient is 


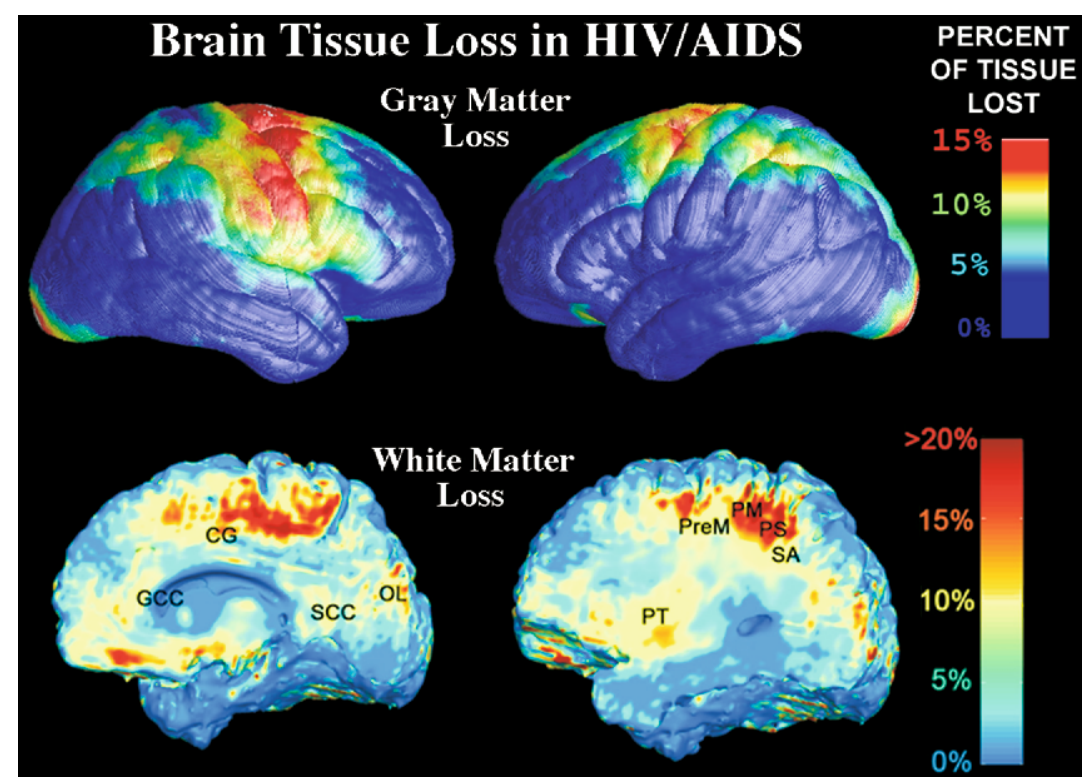

FIG. 7. These two cross-sectional MRI studies used the cortical pattern-matching technique ${ }^{86}$ (top) and the tensor-based mapping (TBM) technique ${ }^{53}$ (bottom) to compare the cortical thickness and subcortical white matter volumes between 26 patients with HIV/AIDS and 14 normal control subjects. Despite aggressive antiretroviral therapy, the cortical maps revealed $15 \%$ atrophy of the primary sensorimotor and the premotor cortices, while TBM showed more than $20 \%$ loss of the adjacent subcortical white matter in the HIV/AIDS subjects relative to the control group. Both the cortical and white matter changes linked to information processing speed and CD4 ${ }^{+}$T-cell counts.

subjected to an invasive neurosurgical procedure. Brain mapping has therefore been increasingly relied upon in clinical decision making.

The 3D hippocampal maps of seizure-free versus nonseizure-free subjects who underwent temporal lobectomy for MTLE revealed that more severe hippocampal atrophy ipsilateral to the preoperative seizure origin and contralateral anterior and lateral hippocampal involvement carried a poorer prognosis with respect to a seizurefree post-surgical outcome. ${ }^{85} \mathrm{~A}$ surface-based cortical thickness study analyzed the preoperative MRI scans of seizure-free MTLE patients who underwent anteromesial temporal lobectomy in comparison to age-matched controls. The patient group showed cortical thinning in the frontal poles and frontal opercular, orbitofrontal, lateral temporal, and occipital areas. Patients with a longer duration of illness showed greater atrophy of the superior frontal and parahippocampal gyri ipsilaterally. ${ }^{85}$

The human immunodeficiency virus (HIV) is readily detected in brain tissue of seropositive patients. Sometimes the infection is latent and patients are asymptomatic; at other times patients may suffer from any or all of dementia, encephalopathy, demyelination, Parkinsonism or other movement disorders, sleep abnormalities, and opportunistic CNS infections. Using the cortical patternmatching technique, Thompson et al. ${ }^{86}$ investigated the changes in cortical thickness in 26 nondemented HIV and 14 control patients (FIG. 7, top). Despite aggressive retroviral therapy, the cortical maps revealed $15 \%$ atrophy of the primary sensorimotor and the premotor cor- tices in HIV subjects relative to the control group. Focal atrophy of the frontopolar and perisylvian regions correlated with the $\mathrm{CD} 4{ }^{+}$T-lymphocyte counts, while prefrontal and parietal cortical thinning correlated with psychomotor slowing and motor deficits.

TBM analyses of the same data (FIG. 7, bottom) extended these findings by reporting white matter degenerative changes in regions underlying the primary and association sensorimotor cortices. These changes were likewise linked to information processing speed and $\mathrm{CD}^{+}{ }^{+} \mathrm{T}$-cell counts, a measure of immune system integrity. ${ }^{53} \mathrm{~A}$ third publication assessed callosal and ventricular changes in a slightly larger HIV-infected group $(n=30)$ against 21 control subjects and reported anterior callosal thinning and frontal ventricular horn enlargement in the seropositive group. Once again, the structural changes (e.g., ventricular expansion) were linked to the $\mathrm{CD} 4^{+}$count and cognitive change. ${ }^{87}$

\section{BRAIN MAPPING IN PSYCHIATRIC DISORDERS}

Schizophrenia is a chronic psychiatric disorder manifesting with episodic psychotic disturbances and progressive pervasive social, cognitive, and functional deterioration. Neuroimaging data have shown the toll of the disease on brain structure to be profound, although there is disagreement regarding its cellular or molecular basis. Schizophrenic patients show hippocampal atrophy ${ }^{88}$ mapping to the anterior and mid-portions of the CA1 and 
CA2 subfields. ${ }^{89}$ This predominantly anterior hippocampal atrophy pattern has been independently replicated. ${ }^{90,91}$ The disease effect may be lateralized in twins, with the left hippocampus being more affected in dizygotic and less affected in monozygotic schizophrenic twins relative to their unaffected siblings. ${ }^{92}$ Cortical thickness studies have revealed atrophy of the frontal, parietal, temporal, and occipital cortices. ${ }^{93,94}$ Current brain mapping efforts in schizophrenia focus on determining whether these cortical changes are modulated by antipsychotic treatment, ${ }^{95}$ and to what extent they occur in the prodromal phase of the illness, ${ }^{96}$ when medications may resist or delay onset of psychosis.

The presence of late-life depression (illness onset after the age of 60) has been linked to bilateral temporal and parietal atrophy. ${ }^{97}$ One TBM study of adults with bipolar disorder demonstrated focal deficits in the orbitofrontal and temporal regions, as well as the amygdala. ${ }^{98}$

In child psychiatry, studies of attention-deficit hyperactivity disorder have shown an anteroposterior dichotomy, with less dorsal prefrontal and anterior temporal and more posterior temporal and inferior parietal cortical gray matter. ${ }^{99}$ Children who subsequently evolve to develop bipolar disorder were reported to have increased left temporal and decreased anterior cingulate cortical thickness. ${ }^{100}$

\section{BRAIN MAPPING OF ABNORMAL DEVELOPMENT IN EARLY LIFE}

Abnormal brain development and stalled or disturbed brain growth can occur in utero and in early childhood from genetic predisposition, infection, or toxic influences. Fetal alcohol syndrome, diagnosed in infants who suffered heavy prenatal alcohol exposure, presents with poor brain and somatic growth, brain and facial malformations and mental retardation. Sowell et al. ${ }^{101}$ demonstrated that the children with fetal alcohol syndrome had $15 \%$ gray matter excess in the perisylvian cortices, suggestive of aberrant regional cortical development. ${ }^{101}$

Williams syndrome is a rare genetic disorder caused by a heterozygous deletion of chromosome 7q11.23. Phenotypically, affected children commonly display dysmorphic facial features, congenital heart and renal malformations, and mild mental retardation with a characteristic sparing of visuospatial functions relative to language and memory faculties. The structural brain derangements in Williams syndrome include increased gyrification in temporoparietal regions. Despite the significantly smaller brain volume, and lower gray and white matter volumes overall, these children had thicker gray matter in the perisylvian regions. This is thought to result from aberrations in cell migration proteins, such as elastin, during early gyrogenesis. ${ }^{102}$

\section{CONCLUSION}

Overall, brain mapping techniques are providing substantial new insights into the trajectory of neurodegenerative disease, the factors that resist or promote disease onset, and the linkage between cognitive decline and regionally specific changes in brain structure and function. Subjects with MCI, in particular, are at fivefold increased risk for conversion to $\mathrm{AD}$ and are therefore the focus of many clinical trials to delay or modulate disease progression. Because of this focus, brain mapping has been used to identify quantitative features of hippocampal and cortical anatomy that are altered in MCI, including discrete changes that predict conversion or link with subtle changes in specific cognitive domains.

At the same time, rapid developments in the imaging techniques themselves are driving research into the molecular hallmarks of AD. In this review, we have focused on MRI scanning, and methods to analyze structural images of the brain, but new PET radiotracers now show great promise as in vivo markers of NFT and amyloid pathology. ${ }^{103}$ The computational anatomy techniques described here are likely to be of great value in establishing the trajectory of these biomarkers as the disease spreads throughout the cortex. ${ }^{104}$

Fusion of different brain mapping modalities with postmortem data is also required to fully relate pathology to structural deterioration, and may help in defining the success of cholinergic or neuroprotective therapies. Ongoing developments in high-field MRI (at field strengths of $7 \mathrm{~T}$ and higher) and in diffusion tensor imaging now reveal the integrity and connectivity of the white matter in unprecedented detail. These imaging approaches bring new sources of power for detecting degenerative illness, and are likely to be invaluable in understanding the impact of neurotherapeutic agents to delay or revert disease progression.

Acknowledgments: This work was generously supported by National Institutes of Health (NIH) National Institute on Aging (NIA) grant K23 AG026803 to L.G.A. (jointly sponsored by the NIA, the American Federation for Aging Research, the John A. Hartford Foundation, the Atlantic Philanthropies, the Starr Foundation, and an anonymous donor); by NIA center grant P50 AG16570 (to L.G.A. and P.M.T.); and by the following NIH grants to P.M.T.: National Institute of Biomedical Imaging and Bioengineering (NIBIB) EB01651; National Library of Medicine (NLM) LM05639; National Center for Research Resources (NCRR) RR019771; National Institute of Mental Health (NIMH) R01 MH071940; NCRR P41 RR013642; and NIH Interdisciplinary Research Consortium U54 RR021813.

\section{REFERENCES}

1. Brodmann $\mathrm{K}$. On the comparative localization of the cortex [Translated from Brodmann K. Vergleichende Lokalizationslehre der Grosshirnrinde in ihren Prinzipien dargestellt auf Grund des 
Zellenbaues. Leipzig: Barth, 1909.]. Springfield, IL: Thomas, 1960.

2. Toga AW, Thompson PM, Mori S, et al. Towards multimodal atlases of the human brain. Nat Rev Neurosci 2006;7:952-966.

3. Monien BH, Apostolova LG, Bitan G. Early diagnostics and therapeutics for Alzheimer's disease: how early can we get there? Expert Rev Neurother 2006;6:1293-1306.

4. Schumock GT. Economic considerations in the treatment and management of Alzheimer's disease. Am J Health Syst Pharm 1998;55 Suppl 2:S17-S21.

5. Petersen RC, Doody R, Kurz A, et al. Current concepts in mild cognitive impairment. Arch Neurol 2001;58:1985-1992.

6. Duyckaerts C, Dickson DW. Neuropathology of Alzheimer's disease. In: Dickson DW, editor. Neurodegeneration: the molecular pathology of dementia and movement disorders. Basel: International Society of Neuropathology, 2003:47-65.

7. Mesulam MM. Aging, Alzheimer's disease and dementia. In: Mesulam MM, editor. Principles of behavioral and cognitive neurology, 2nd ed. Oxford: Oxford University Press, 2000:439510.

8. Hogan RE, Mark KE, Wang L, Joshi S, Miller MI, Bucholz RD. Mesial temporal sclerosis and temporal lobe epilepsy: MR imaging deformation-based segmentation of the hippocampus in five patients. Radiology 2000;216:291-297.

9. Yushkevich PA, Piven J, Hazlett HC, et al. User-guided 3D active contour segmentation of anatomical structures: significantly improved efficiency and reliability. Neuroimage 2006;31: $1116-1128$.

10. Pitiot A, Delingette H, Thompson PM, Ayache N. Expert knowledge-guided segmentation system for brain MRI. Neuroimage 2004;23 Suppl 1:S85-S96.

11. Jack CR Jr, Petersen RC, Xu Y, et al. Rates of hippocampal atrophy correlate with change in clinical status in aging and AD. Neurology 2000;55:484-489.

12. Jack CR Jr, Petersen RC, Xu Y, et al. Rate of medial temporal lobe atrophy in typical aging and Alzheimer's disease. Neurology 1998;51:993-999.

13. Jack CR Jr, Shiung MM, Gunter JL, et al. Comparison of different MRI brain atrophy rate measures with clinical disease progression in AD. Neurology 2004;62:591-600.

14. Ridha BH, Barnes J, Bartlett JW, et al. Tracking atrophy progression in familial Alzheimer's disease: a serial MRI study. Lancet Neurol 2006;5:828-834.

15. Schonheit B, Zarski R, Ohm TG. Spatial and temporal relationships between plaques and tangles in Alzheimer-pathology. Neurobiol Aging 2004;25:697-711.

16. Apostolova LG, Dinov ID, Dutton RA, et al. 3D comparison of hippocampal atrophy in amnestic mild cognitive impairment and Alzheimer's disease. Brain 2006;129:2867-2873.

17. Apostolova LG, Dutton RA, Dinov ID, et al. Conversion of mild cognitive impairment to Alzheimer disease predicted by hippocampal atrophy maps. Arch Neurol 2006;63:693-699.

18. deToledo-Morrell L, Stoub TR, Bulgakova M, et al. MRI-derived entorhinal volume is a good predictor of conversion from MCI to AD. Neurobiol Aging 2004;25:1197-1203.

19. Du AT, Schuff N, Amend D, et al. Magnetic resonance imaging of the entorhinal cortex and hippocampus in mild cognitive impairment and Alzheimer's disease. J Neurol Neurosurg Psychiatry 2001;71:441-447.

20. Xu Y, Jack CR Jr, O'Brien PC, et al. Usefulness of MRI measures of entorhinal cortex versus hippocampus in AD. Neurology 2000; 54:1760-1767.

21. Dickerson BC, Goncharova I, Sullivan MP, et al. MRI-derived entorhinal and hippocampal atrophy in incipient and very mild Alzheimer's disease. Neurobiol Aging 2001;22:747-754.

22. Du AT, Schuff N, Kramer JH, et al. Higher atrophy rate of entorhinal cortex than hippocampus in AD. Neurology 2004;62: $422-427$.

23. Wright IC, McGuire PK, Poline JB, et al. A voxel-based method for the statistical analysis of gray and white matter density applied to schizophrenia. Neuroimage 1995;2:244-252.

24. Ashburner J, Friston KJ. Voxel-based morphometry: the methods. Neuroimage 2000;11:805-821.
25. Ashburner J, Friston KJ. Why voxel-based morphometry should be used. Neuroimage 2001;14:1238-1243.

26. Friston KJ, Holmes AP, Worsley KJ, Poline JP, Frith CD, Frackowiak RSJ. Statistical parametric maps in functional imaging: a general linear approach. Hum Brain Mapp 1995;2:189-210.

27. Good CD, Ashburner J, Frackowiak RS. Computational neuroanatomy: new perspectives for neuroradiology. Rev Neurol (Paris) 2001;157:797-806.

28. Good CD, Johnsrude IS, Ashburner J, Henson RN, Friston KJ, Frackowiak RSJ. A voxel-based morphometric study of ageing in 465 normal adult human brains. Neuroimage 2001;14:21-36.

29. Bookstein FL. "Voxel-based morphometry" should not be used with imperfectly registered images. Neuroimage 2001;14:14541462.

30. Thacker N. Tutorial: a critical analysis of voxel based morphometry (VBM). Division of Imaging Science and Biomedical Engineering, University of Manchester. Updated Aug. 29, 2005. Accessed March 3, 2007. Available at: http://www.tina-vision.net/ docs/memos/2003-011.pdf.

31. Davatzikos C, Genc A, Xu D, Resnick SM. Voxel-based morphometry using the RAVENS maps: methods and validation using simulated longitudinal atrophy. Neuroimage 2001;14:13611369.

32. Salmond CH, Ashburner J, Vargha-Khadem F, Connelly A, Gadian DG, Friston KJ. Distributional assumptions in voxel-based morphometry. Neuroimage 2002;17:1027-1030.

33. Baron JC, Chetelat G, Desgranges B, et al. In vivo mapping of gray matter loss with voxel-based morphometry in mild Alzheimer's disease. Neuroimage 2001;14:298-309.

34. Busatto GF, Garrido GE, Almeida OP, et al. A voxel-based morphometry study of temporal lobe gray matter reductions in Alzheimer's disease. Neurobiol Aging 2003;24:221-231.

35. Scahill RI, Schott JM, Stevens JM, et al. Mapping the evolution of regional atrophy in Alzheimer's disease: unbiased analysis of fluid-registered serial MRI. Proc Natl Acad Sci U S A 2002;99: 4703-4707.

36. Good CD, Scahill RI, Fox NC, et al. Automatic differentiation of anatomical patterns in the human brain: validation with studies of degenerative dementias. Neuroimage 2002;17:29-46.

37. Bozzali M, Filippi M, Magnani G, et al. The contribution of voxel-based morphometry in staging patients with mild cognitive impairment. Neurology 2006;67:453-460.

38. Chetelat G, Desgranges B, De La Sayette V, Viader F, Eustache F, Baron JC. Mapping gray matter loss with voxel-based morphometry in mild cognitive impairment. Neuroreport 2002;13: 1939-1943.

39. Karas GB, Scheltens P, Rombouts SA, et al. Global and local gray matter loss in mild cognitive impairment and Alzheimer's disease. Neuroimage 2004;23:708-716.

40. Chetelat G, Landeau B, Eustache F, et al. Using voxel-based morphometry to map the structural changes associated with rapid conversion in MCI: a longitudinal MRI study. Neuroimage 2005; 27:934-946.

41. Fox NC, Warrington EK, Rossor MN. Serial magnetic resonance imaging of cerebral atrophy in preclinical Alzheimer's disease. Lancet 1999;353:2125.

42. Carmichael OT, Kuller LH, Lopez OL, et al. Ventricular volume and dementia progression in the Cardiovascular Health Study. Neurobiol Aging 2007;28:389-397.

43. Sowell ER, Thompson PM, Holmes CJ, Jernigan TL, Toga AW. In vivo evidence for post-adolescent brain maturation in frontal and striatal regions. Nat Neurosci 1999;2:859-861.

44. Maguire EA, Gadian DG, Johnsrude IS, et al. Navigation-related structural change in the hippocampi of taxi drivers. Proc Natl Acad Sci U S A 2000;97:4398-4403.

45. Rosen HJ, Allison SC, Schauer GF, Gorno-Tempini ML, Weiner MW, Miller BL. Neuroanatomical correlates of behavioural disorders in dementia. Brain 2005;128:2612-2625.

46. Mummery CJ, Patterson K, Price CJ, Ashburner J, Frackowiak RSJ, Hodges JR. A voxel-based morphometry study of semantic dementia: relationship between temporal lobe atrophy and semantic memory. Ann Neurol 2000;47:36-45. 
47. O'Brien JT, Paling S, Barber R, et al. Progressive brain atrophy on serial MRI in dementia with Lewy bodies, AD, and vascular dementia. Neurology 2001;56:1386-1388.

48. Whitwell JL, Weigand SD, Shiung MM, et al. Focal atrophy in dementia with Lewy bodies on MRI: a distinct pattern from Alzheimer's disease. Brain 2007;130:708-719.

49. Gitelman DR, Ashburner J, Friston KJ, Tyler LK, Price CJ. Voxel-based morphometry of herpes simplex encephalitis. Neuroimage 2001;13:623-631.

50. Fischl B, Sereno MI, Dale AM. Cortical surface-based analysis. II: Inflation, flattening, and a surface-based coordinate system. Neuroimage 1999;9:195-207.

51. Thompson PM, Woods RP, Mega MS, Toga AW. Mathematical/ computational challenges in creating deformable and probabilistic atlases of the human brain. Hum Brain Mapp 2000;9:81-92.

52. Gee JC, Thompson PM. Guest editorial: Special issue on computational neuroanatomy. IEEE Trans Med Imaging 2007;26: $425-426$.

53. Chiang MC, Dutton RA, Hayashi KM, et al. 3D pattern of brain atrophy in HIV/AIDS visualized using tensor-based morphometry. Neuroimage 2007;34:44-60.

54. Fox NC, Cousens S, Scahill R, Harvey RJ, Rossor MN. Using serial registered brain magnetic resonance imaging to measure disease progression in Alzheimer disease: power calculations and estimates of sample size to detect treatment effects. Arch Neurol 2000;57:339-344.

55. Leow AD, Klunder AD, Jack CR Jr, et al. Longitudinal stability of MRI for mapping brain change using tensor-based morphometry. Neuroimage 2006;31:627-640.

56. Lee $\mathrm{AD}$, Leow $\mathrm{AD}$, Lu A, et al. 3D pattern of brain abnormalities in fragile $\mathrm{X}$ syndrome visualized using tensor-based morphometry. Neuroimage 2007;34:924-938.

57. Chiang MC, Reiss AL, Lee AD, et al. 3D pattern of brain abnormalities in Williams syndrome visualized using tensor-based morphometry. Neuroimage 2007 [Epub in advance of print April 20, 2007]

58. Lepore N, Brun CA, Chou YY, et al. Generalized tensor-based morphometry of HIV/AIDS using multivariate statistics on strain matrices and their application to HIV/AIDS. IEEE Trans Med Imaging 2007 (in press).

59. Thompson PM, Hayashi KM, de Zubicaray GI, et al. Mapping hippocampal and ventricular change in Alzheimer disease. Neuroimage 2004;22:1754-1766

60. Csernansky JG, Wang L, Joshi S, et al. Early DAT [dementia of the Alzheimer type] is distinguished from aging by high-dimensional mapping of the hippocampus. Neurology 2000;55:16361643.

61. Chou Y, Lepore N, Zubiricay GI, et al. Automated 3D mapping and shape analysis of the lateral ventricles via fluid registration of multiple surface-based atlases. IEEE 2007 International Symposium on Biomedical Imaging, April 12-15, 2007, Arlington, VA.

62. Zeineh MM, Engel SA, Thompson PM, Bookheimer SY. Dynamics of the hippocampus during encoding and retrieval of facename pairs. Science 2003;299:577-580.

63. Mueller SG, Stables L, Du AT, et al. Measurement of hippocampal subfields and age-related changes with high resolution MRI at 4T. Neurobiol Aging 2007;28:719-726.

64. Augustinack JC, van der Kouwe AJ, Blackwell ML, et al. Detection of entorhinal layer II using 7 tesla [corrected] magnetic resonance imaging. Ann Neurol 2005;57:489-494 [Erratum in: Ann Neurol 2005;58:172].

65. Frisoni GB, Sabattoli F, Lee AD, Dutton RA, Toga AW, Thompson PM. In vivo neuropathology of the hippocampal formation in AD: a radial mapping MR-based study. Neuroimage 2006;32: $104-110$.

66. Becker JT, Davis SW, Hayashi KM, et al. Three-dimensional patterns of hippocampal atrophy in mild cognitive impairment. Arch Neurol 2006;63:97-101.

67. Wang L, Swank JS, Glick IE, et al. Changes in hippocampal volume and shape across time distinguish dementia of the Alzheimer type from healthy aging. Neuroimage 2003;20:667-682.

68. Roybal DJ, Dutton RA, Hayashi KM, et al. Mapping ApoE4 and gender effects on hippocampal atrophic rates: a longitudinal MRI study of normal aging. Poster presentation. 2005 Annual Scientific Meeting of the American Geriatric Society, Orlando, FL, 2005.

69. Bookheimer SY, Braskie MN, Burggren AC, et al. Functional MRI and structure-function mapping in the early diagnosis of Alzheimer's disease. Annual Meeting of the American College of Neuropsychopharmacology, Hollywood, FL, 2006.

70. Thompson PM, Hayashi KM, de Zubicaray G, et al. Dynamics of gray matter loss in Alzheimer's disease. J Neurosci 2003;23:9941005.

71. Apostolova LG, Steiner CA, Akopyan GG, et al. 3D gray matter atrophy mapping in mild cognitive impairment and mild Alzheimer's disease. Arch Neurol 2007 (in press).

72. Lerch JP, Pruessner JC, Zijdenbos A, Hampel H, Teipel SJ, Evans AC. Focal decline of cortical thickness in Alzheimer's disease identified by computational neuroanatomy. Cereb Cortex 2005; 15:995-1001.

73. Singh V, Chertkow H, Lerch JP, Evans AC, Dorr AE, Kabani NJ. Spatial patterns of cortical thinning in mild cognitive impairment and Alzheimer's disease. Brain 2006;129:2885-2893.

74. Frisoni GB, Pievani M, Testa C, et al. The topography of grey matter involvement in early and late onset Alzheimer's disease. Brain 2007;130:720-730.

75. Apostolova LG, Lu PH, Rogers S, et al. 3D mapping of minimental state examination performance in clinical and preclinical Alzheimer disease. Alzheimer Dis Assoc Disord 2006;20:224 231.

76. Thompson PM, Hayashi KM, Sowell ER, et al. Mapping cortical change in Alzheimer's disease, brain development, and schizophrenia. Neuroimage 2004;23 Suppl 1:S2-S18.

77. Apostolova LG, Lu P, Rogers S, et al. 3D mapping of language networks in clinical and pre-clinical Alzheimer's disease. Brain Lang 2007 [Epub in advance of print May 7, 2007].

78. Apostolova LG, Lu PH, Rogers S, et al. 3D mapping of verbal memory performance in clinical and pre-clinical Alzheimer's disease. Poster presentation. 131st Annual Meeting of the American Neurological Association, Chicago, IL, 2006.

79. Bernstein MA, Lin C, Borowski BJ, et al. Alzheimer's Disease Neuroimaging Initiative (ADNI): the MR imaging protocol Poster presentation. State-of-the-art cardiovascular and neuro MRI: a joint workshop of the ISMRM and CSR, Beijing, China, 2005.

80. Ballmaier M, O'Brien JT, Burton EJ, et al. Comparing gray matter loss profiles between dementia with Lewy bodies and Alzheimer's disease using cortical pattern matching: diagnosis and gender effects. Neuroimage 2004;23:325-335.

81. Perry RJ, Graham A, Williams G, et al. Patterns of frontal lobe atrophy in frontotemporal dementia: a volumetric MRI study. Dement Geriatr Cogn Disord 2006;22:278-287.

82. Gorno-Tempini ML, Dronkers NF, Rankin KP, et al. Cognition and anatomy in three variants of primary progressive aphasia. Ann Neurol 2004;55:335-346.

83. Apostolova LG, Clark DG, Zoumalan CI, et al. 3D mapping of gray matter atrophy in semantic dementia and frontal variant frontotemporal dementia. Poster presentation. 10th International Conference of Alzheimer's Disease and Related Disorders, Madrid, Spain, 2006.

84. Du AT, Schuff N, Rosen HJ, et al. Cortical thinning in Alzheimer's disease and frontotemporal dementia assessed using MRI. Poster presentation. 58th Annual American Academy of Neurology Meeting, San Diego, CA, 2006.

85. Lin JJ, Salamon N, Dutton RA, et al. Three-dimensional preoperative maps of hippocampal atrophy predict surgical outcomes in temporal lobe epilepsy. Neurology 2005;65:1094-1097.

86. Thompson PM, Dutton RA, Hayashi KM, et al. Thinning of the cerebral cortex visualized in HIV/AIDS reflects $\mathrm{CD}^{+}{ }^{+} \mathrm{T}$ lymphocyte decline. Proc Natl Acad Sci U S A 2005;102:1564715652 .

87. Thompson PM, Dutton RA, Hayashi KM, et al. 3D mapping of ventricular and corpus callosum abnormalities in HIV/AIDS. Neuroimage 2006;31:12-23. 
88. Narr KL, Thompson PM, Sharma T, et al. Three-dimensional mapping of temporo-limbic regions and the lateral ventricles in schizophrenia: gender effects. Biol Psychiatry 2001;50:84-97.

89. Narr KL, Thompson PM, Szeszko P, et al. Regional specificity of hippocampal volume reductions in first-episode schizophrenia. Neuroimage 2004;21:1563-1575.

90. Csernansky JG, Joshi S, Wang L, et al. Hippocampal morphometry in schizophrenia by high dimensional brain mapping. Proc Natl Acad Sci U S A 1998;95:11406-11411.

91. Csernansky JG, Wang L, Jones D, et al. Hippocampal deformities in schizophrenia characterized by high dimensional brain mapping. Am J Psychiatry 2002;159:2000-2006.

92. Narr KL, van Erp TG, Cannon TD, et al. A twin study of genetic contributions to hippocampal morphology in schizophrenia. Neurobiol Dis 2002;11:83-95.

93. Narr KL, Bilder RM, Toga AW, et al. Mapping cortical thickness and gray matter concentration in first episode schizophrenia. Cereb Cortex 2005;15:708-719.

94. Narr KL, Toga AW, Szeszko P, et al. Cortical thinning in cingulate and occipital cortices in first episode schizophrenia. Biol Psychiatry 2005;58:32-40.

95. Thompson PM. Time-lapse mapping of brain changes in schizophrenia and over the human lifespan. Int J Neuropsychopharmacol 2006;9(suppl):S34

96. Sun D, Phillips L, Velakoulis D, et al. Progressive brain structural changes mapped as psychosis develops in "at risk" individuals. Poster presentation. International Congress on Schizophrenia Research, Colorado Springs, CO, 2007.

97. Ballmaier M, Kumar A, Thompson PM, et al. Localizing gray matter deficits in late-onset depression using computational cortical pattern matching methods. Am J Psychiatry 2004;161:2091-2099.

98. Foland LC, Altshuler LL, Leow AD, et al. A tensor-based morphometric study of bipolar disorder. Poster presentation. 12th Annual OHBM Meeting, Florence, Italy, 2006.

99. Sowell ER, Thompson PM, Welcome SE, et al. Cortical abnormalities in children and adolescents with attention-deficit hyperactivity disorder. Lancet 2003;362:1699-1707.

100. Gogtay N, Ordonez A, Herman D, et al. Dynamic mapping of cortical development before and after the onset of pediatric bipolar illness. J Neurol Neurosurg Psychiatry 2007 (in press).

101. Sowell ER, Thompson PM, Peterson BS, et al. Mapping cortical gray matter asymmetry patterns in adolescents with heavy prenatal alcohol exposure. Neuroimage 2002;17:1807-1819.

102. Thompson PM, Lee AD, Dutton RA, et al. Abnormal cortical complexity and thickness profiles mapped in Williams syndrome. J Neurosci 2005;25:4146-4158.

103. Small GW, Kepe V, Ercoli LM, et al. PET of brain amyloid and tau in mild cognitive impairment. N Engl J Med 2006;355:26522663.

104. Small GW, Protas HD, Huang SC, et al. FDDNP binding values from cortical hemispheric surface maps correlate with MMSE scores. Poster presentation. Alzheimer's Association International Conference on the Prevention of Dementia. Washington, DC, 2007.

105. Duvernoy HM. The human hippocampus: an atlas of applied anatomy. Munich: Bergmann Verlag, 1988:77-95.

106. West MJ, Gundersen HJ. Unbiased stereological estimation of the number of neurons in the human hippocampus. J Comp Neurol 1990;296:1-22. 